\title{
ON THE SIZE OF LEMNISCATES OF POLYNOMIALS IN ONE AND SEVERAL VARIABLES
}

\author{
A. CUYT, K. DRIVER, AND D. S. LUBINSKY
}

(Communicated by J. Marshall Ash)

\begin{abstract}
In the convergence theory of rational interpolation and Padé approximation, it is essential to estimate the size of the lemniscatic set $E:=$ $\left\{z:|z| \leq r\right.$ and $\left.|P(z)| \leq \epsilon^{n}\right\}$, for a polynomial $P$ of degree $\leq n$. Usually, $P$ is taken to be monic, and either Cartan's Lemma or potential theory is used to estimate the size of $E$, in terms of Hausdorff contents, planar Lebesgue measure $m_{2}$, or logarithmic capacity cap. Here we normalize $\|P\|_{L_{\infty}(|z| \leq r)}=1$ and show that $\operatorname{cap}(E) \leq 2 r \epsilon$ and $m_{2}(E) \leq \pi(2 r \epsilon)^{2}$ are the sharp estimates for the size of $E$. Our main result, however, involves generalizations of this to polynomials in several variables, as measured by Lebesgue measure on $\mathbb{C}^{n}$ or product capacity and Favarov's capacity. Several of our estimates are sharp with respect to order in $r$ and $\epsilon$.
\end{abstract}

\section{$\S 1$. INTRODUCTION}

In the convergence theory of Padé approximation, and more generally rational interpolation, an essential ingredient is an estimate on the size of the lemniscate

$$
E(P ; \epsilon):=\left\{z:|P(z)| \leq \epsilon^{n}\right\},
$$

where $P$ is a polynomial of degree $\leq n$. There are several ways to provide this estimate. Cartan's Lemma shows that if $P$ is normalized to be monic of degree $n$, then we can cover this set by a union of $\ell \leq n$ balls $B_{j}, 1 \leq j \leq \ell$, whose diameters $d\left(B_{j}\right)$ satisfy, for a given $\alpha>0$,

$$
\sum_{j=1}^{\ell}\left(d\left(B_{j}\right)\right)^{\alpha} \leq e 4^{\alpha} \epsilon^{\alpha} .
$$

The remarkable thing about the estimate is its independence of the degree of $P$. See [1, p. 194], [7], [9], [12], [14] for further details and extensions. As far as we know, the sharp constant (that should replace $e 4^{\alpha}$ ) in Cartan's Lemma is still an unsolved problem. The authors thank Peter Borwein for informing them that the conjectured sharp constant for $\alpha=1$ is 4 .

Received by the editors September 19, 1994 and, in revised form, January 30, 1995.

1991 Mathematics Subject Classification. Primary 30C10, 32A30, 41A10, 41A21.

Key words and phrases. Polynomials, several complex variables, logarithmic capacity, product capacities, lemniscates, potential theory, Favarov's capacity. 
An even more appropriate set function to measure $E(P ; \epsilon)$ for monic $P$ is $\log$ arithmic capacity. Amongst the many equivalent definitions, we mention the one involving the Chebyshev constant: For compact $F \subset \mathbb{C}$,

$$
\operatorname{cap}(F):=\lim _{n \rightarrow \infty}\left[\min \left\{\|P\|_{L_{\infty}(F)}: P \text { monic of degree } n\right\}\right]^{1 / n} .
$$

See [7], [9], [12]. Here we have the identity

$$
\operatorname{cap}(E(P ; \epsilon))=\epsilon .
$$

In applications of these to Padé approximation, one usually has to estimate

$$
\|P\|_{L_{\infty}(|t|=r)} /|P(z)|
$$

where $|z|<r$ lies outside some exceptional set. Normalizing $P$ to be monic helps us to estimate the denominator in (1.4), but then zeros of $P$ of large modulus are troublesome in estimating the numerator. To circumvent this, researchers in Padé approximation such as Nuttall, Pommerenke, Goncar, and others [8], [13], [15] split the zeros of $P$ into sets $\left\{u_{j}:\left|u_{j}\right| \leq 2 r\right\}$ and $\left\{v_{j}:\left|v_{j}\right|>2 r\right\}$ and normalized $P$ as

$$
P(z)=\prod_{j}\left(z-u_{j}\right) \prod_{j}\left(1-z / v_{j}\right) .
$$

Since for $|z| \leq r$,

$$
\frac{1}{2}<\left|1-z / v_{j}\right|<\frac{3}{2} ; \quad\left|z-u_{j}\right| \leq 3 r
$$

we easily see that

$$
\|P\|_{L_{\infty}(|t|=r)} /|P(z)| \leq(3 \max \{1, r\})^{n} /\left|\prod_{j}\left(z-u_{j}\right)\right|
$$

and now the size of the exceptional set can be estimated by (1.2) or (1.3).

In studying convergence theory of Padé approximants of several variables [5], [8], [11], one can try to extend this approach to several variable polynomials $P\left(z_{1}, z_{2}, \ldots, z_{\ell}\right)$. One can fix $z_{2}, z_{3}, \ldots, z_{\ell}$ and then factorize as above in terms of $z_{1}$. However the $u_{j}$ and $v_{j}$ depend in a complicated way (implicit function theorem, etc.) on the other variables $z_{j}, 2 \leq j \leq \ell$, and normalization becomes a real problem.

So we found it desirable to instead normalize

$$
\|P\|_{L_{\infty}(|z|=r)}=1
$$

and study directly the size of

$$
E(P ; r ; \epsilon):=\left\{z:|z| \leq r \text { and }|P(z)| \leq \epsilon^{n}\right\}
$$


in the hope of producing an approach that will more easily extend to polynomials in several variables. Of course, this normalization avoids having to separate zeros of $P$ into large and small modulus when we estimate the ratio (1.4).

Let $m_{2}$ denote planar Lebesgue measure and, for $\alpha>0$, let $h_{\alpha}$ denote $\alpha$ dimensional Hausdorff content, so that

$$
h_{\alpha}(E):=\inf \left\{\sum_{j=1}^{\infty}\left(d\left(B_{j}\right)\right)^{\alpha}:\left\{B_{j}\right\} \text { are balls with } E \subset \bigcup_{j=1}^{\infty} B_{j}\right\} .
$$

Here $d\left(B_{j}\right)$ denotes the diameter of $B_{j}$. Of course, for measurable $E$,

$$
m_{2}(E)=\frac{\pi}{4} h_{2}(E)
$$

The sharp form of (a) of the following simple one-variable result is apparently new:

Theorem 1.1. (a) For polynomials $P$ of degree $\leq n$, normalized by (1.5), and $\epsilon>0$, we have

$$
\begin{aligned}
& \operatorname{cap}(E(P ; r ; \epsilon)) \leq 2 r \epsilon ; \\
& m_{2}(E(P ; r ; \epsilon)) \leq \pi(2 r \epsilon)^{2} .
\end{aligned}
$$

If $L$ is any line in the plane, then

$$
h_{1}(L \cap E(P ; r ; \epsilon)) \leq 8 r \epsilon
$$

Given $n \geq 1$ and $r>0$, (1.8) and (1.9) are sharp in the sense that

$$
\begin{aligned}
& \sup _{P, \epsilon} \operatorname{cap}(E(P ; r ; \epsilon)) / \epsilon=2 r ; \\
& \sup _{P, \epsilon} m_{2}(E(P ; r ; \epsilon)) / \epsilon^{2}=\pi(2 r)^{2} .
\end{aligned}
$$

In each case the sup is taken over $\epsilon>0$ and polynomials $P$ of degree $n$ satisfying (1.5). Moreover, (1.10) is almost sharp in the sense that given $n \geq 1$ and $r>0$,

$$
\sup _{L, P, \epsilon} h_{1}(L \cap E(P ; r ; \epsilon)) / \epsilon \geq 8 r 2^{-1 / n} .
$$

In the last sup, $L$ refers to all lines in $\mathbb{C}$.

(b) Given $\alpha>0$ and $P$ of degree $\leq n$, normalized by (1.5), we have

$$
h_{\alpha}(E(P ; r ; \epsilon)) \leq 18(4 r \epsilon)^{\alpha} .
$$

Of course, (1.10) shows that the diameter of $E(P ; r ; \epsilon)$ is at most $8 r \epsilon$, and our examples that prove (1.13) show this is sharp as $n \rightarrow \infty$. We remark that using Nuttall's method, Pommerenke [15] established the weaker estimate

$$
\operatorname{cap}(E(P ; r ; \epsilon)) \leq 3 r \epsilon .
$$


Our proof of (1.8) involves the Walsh-Bernstein lemma and simple estimates on Green's functions. Then standard inequalities relating $h_{\alpha}$ and $m_{2}$ to cap give (1.9), (1.10), (1.14).

As we have mentioned, our main goal is estimation of the lemniscates of polynomials of several variables. Some intuition is provided by the polynomial

$$
P(z, w):=(z w)^{n} .
$$

We see that given $r \geq \epsilon>0$,

$$
\begin{aligned}
E(P ; r ; \epsilon): & =\left\{(z, w):|z|,|w| \leq r \text { and }|P(z, w)| \leq \epsilon^{n}\right\} \\
& =\{(z, w):|z|,|w| \leq r \text { and }|z w| \leq \epsilon\} \\
& =\bigcup_{|w| \leq r}\{(z, w):|z| \leq \min \{r, \epsilon /|w|\}\} .
\end{aligned}
$$

Then if $m_{4}$ denotes Lebesgue measure on $\mathbb{C}^{2}$, Fubini's theorem gives

$$
\begin{aligned}
m_{4}(E(P ; r ; \epsilon)) & =m_{2} \times m_{2}(E(P ; r ; \epsilon))=\int_{|w| \leq r} \pi \min \{r, \epsilon /|w|\}^{2} d m_{2}(w) \\
& =\pi^{2} \epsilon^{2}\left[1+2 \log \frac{r^{2}}{\epsilon}\right]
\end{aligned}
$$

provided $r^{2} \geq \epsilon$. If $r^{2}<\epsilon$, we obtain instead $\left(\pi r^{2}\right)^{2}$. (We used polar coordinates to compute the integral.) As $r \rightarrow \infty$, the measure of $E(P ; r ; \epsilon) \rightarrow \infty$, which is surprising when one thinks of one variable, for which the measure/content/cap is bounded independent of $r$. If we consider the normalized polynomial

$$
P_{1}(z, w):=\left(z w / r^{2}\right)^{n}
$$

which has

$$
\max _{|z|,|w| \leq r}\left|P_{1}(z, w)\right|=1
$$

then we see that

$$
\begin{aligned}
E\left(P_{1} ; r ; \epsilon\right): & =\left\{(z, w):|z|,|w| \leq r \text { and }\left|P_{1}(z, w)\right| \leq \epsilon^{n}\right\} \\
& =\left\{(z, w):|z|,|w| \leq r \text { and }|z w| \leq\left(\epsilon r^{2}\right)\right\}
\end{aligned}
$$

so we can apply (1.15) if we replace $\epsilon$ there by $\epsilon r^{2}$. Thus if $\epsilon \leq 1$,

$$
m_{4}\left(E\left(P_{1} ; r ; \epsilon\right)\right)=\left(\pi r^{2} \epsilon\right)^{2}\left[1+2 \log \frac{1}{\epsilon}\right] .
$$

(If $\epsilon>1$, it is instead $\left(\pi r^{2}\right)^{2}$.) This simple example shows that our next result has estimates of the correct order in $r$ and $\epsilon$ for 2 dimensions, and for more general 
$k$ dimensions, one can perform analogous calculations with $P\left(z_{1}, z_{2}, \ldots, z_{k}\right):=$ $\left(z_{1} z_{2} \ldots z_{k} / r^{k}\right)^{n}$.

Our two main theorems treat polynomials $P\left(z_{1}, z_{2}, \ldots, z_{k}\right)$ that are of degree $\leq n$ in each variable $z_{j}$ (so that no higher power than $z_{j}^{n}$ appears in $P$ ), $1 \leq j \leq k$, normalized by

$$
\max \left\{\left|P\left(z_{1}, z_{2}, \ldots, z_{k}\right)\right|:\left|z_{j}\right| \leq r, 1 \leq j \leq k\right\}=1 .
$$

We denote its lemniscate by

$E(P ; r ; \epsilon):=\left\{\left(z_{1}, z_{2}, \ldots, z_{k}\right):\left|z_{j}\right| \leq r, 1 \leq j \leq k\right.$, and $\left.\left|P\left(z_{1}, z_{2}, \ldots, z_{k}\right)\right| \leq \epsilon^{n}\right\}$.

Let $m_{2 k}$ denote Lebesgue measure on $\mathbb{C}^{k}$ and let $\log _{2}$ denote the log to the base 2 .

Theorem 1.2. For polynomials $P$ that are of degree $\leq n$ in each of their $k$ variables $z_{1}, z_{2}, \ldots, z_{k}$, normalized by (1.20), and for $\epsilon>0$, we have

$$
m_{2 k}(E(P ; r ; \epsilon)) \leq\left(16 \pi r^{2}\right)^{k} \epsilon^{2} \max \left\{1, \log _{2} \frac{2^{k-1}}{\epsilon}\right\}^{k-1} .
$$

We note that the estimate (1.22) remains valid if we replace $=1$ in (1.20) by $\geq 1$. There is a well-developed theory of capacities in $\mathbb{C}^{n}[3],[6],[17],[18],[20]$, but for our purposes these are difficult to estimate, especially as there is no longer such a simple relationship between potentials and logs of polynomials. We prefer to use product capacity and Favarov's capacity (a close cousin of Ronkin's $\gamma$-capacity), as discussed by Cegrell [6, p.86, p.81].

For compact $E \subset \mathbb{C}^{2}$, we define its product capacity cap ${ }^{(2)}(E)$ by

$$
\operatorname{cap}^{(2)}(E):=\int_{0}^{\infty} \operatorname{cap}\left\{z_{1}: \operatorname{cap}\left\{z_{2}:\left(z_{1}, z_{2}\right) \in E\right\}>s\right\} d s .
$$

More generally, for $E \subset \mathbb{C}^{k}$, we define cap ${ }^{(k)}(E)$ inductively by

$$
\operatorname{cap}^{(k)}(E):=\int_{0}^{\infty} \operatorname{cap}\left\{z_{1}: \operatorname{cap}^{(k-1)}\left\{\left(z_{2}, \ldots, z_{k}\right):\left(z_{1}, z_{2}, \ldots, z_{k}\right) \in E\right\}>s\right\} d s .
$$

This apparently strange definition really does yield a product capacity: If

$$
E=E_{1} \times E_{2} \times \cdots \times E_{k},
$$

where each $E_{j} \subset \mathbb{C}$, then

$$
\operatorname{cap}^{(k)}(E)=\prod_{j=1}^{k} \operatorname{cap} E_{j}
$$

Recall that a unitary transformation $A$ is a $k \times k$ matrix with complex entries such that $\bar{A}^{T} A=I$. Favarov's capacity $\Gamma_{k}^{F}(E)$ of $E \subset \mathbb{C}^{k}$ is defined by [6, p. 93]

$$
\Gamma_{k}^{F}(E)=\sup \left\{\operatorname{cap}^{(k)}(A(E)): A \text { a unitary transformation }\right\} .
$$

We say that a polynomial $P\left(z_{1}, z_{2}, \ldots, z_{k}\right)$ is of total degree $\leq n$, if each term $c z_{1}^{j_{1}} z_{2}^{j_{2}} \ldots z_{k}^{j_{k}}$ in its Maclaurin series has $j_{1}+j_{2}+\cdots+j_{k} \leq n$. 
Theorem 1.3. For polynomials $P$ that are of degree $\leq n$ in each of their $k$ variables $z_{1}, z_{2}, \ldots, z_{k}$, normalized by (1.20), and for $\epsilon>0$, we have

$$
\operatorname{cap}^{(k)}(E(P ; r ; \epsilon)) \leq C_{1} r^{k} \epsilon \max \left\{1, \log _{2} \frac{1}{\epsilon}\right\}^{k-1}
$$

and

$$
\Gamma_{k}^{F}(E(P ; r ; \epsilon)) \leq C_{1} r^{k} \epsilon^{1 / k} \max \left\{1, \log _{2} \frac{1}{\epsilon}\right\}^{k-1}
$$

Here $C_{1}$ is independent of $r, P, \epsilon, n$. If in addition $P$ is of total degree $\leq n$, then

$$
\Gamma_{k}^{F}(E(P ; r ; \epsilon)) \leq C_{1} r^{k} \epsilon \max \left\{1, \log _{2} \frac{1}{\epsilon}\right\}^{k-1} .
$$

The estimate (1.26) is sharp with respect to order in $\epsilon$ and $r$. For simplicity, consider $k=2$ and $P_{1}$ of (1.16), and recall (1.17), (1.18). Now for fixed $z$,

$$
\operatorname{cap}\left\{w:|w| \leq r \text { and }|w| \leq \epsilon r^{2} /|z|\right\}=r \min \{1, \epsilon r /|z|\},
$$

and hence, if $\epsilon \leq 1$,

$$
\begin{aligned}
\operatorname{cap}^{(2)}\left(E\left(P_{1} ; r ; \epsilon\right)\right) & =\int_{0}^{\infty} \operatorname{cap}\{z:|z| \leq r \text { and } r \min \{1, \epsilon r /|z|\}>s\} d s \\
& =r \int_{0}^{r} \min \{1, \epsilon r / s\} d s=r^{2} \epsilon\left[1+\log \frac{1}{\epsilon}\right] .
\end{aligned}
$$

We prove Theorem 1.1 in Section 2, and Theorems 1.2 and 1.3 in Section 3.

\section{§2. Proof of Theorem 1.1}

We recall that if $E$ is a compact set with cap $E>0$ and connected complement, then its Green function with pole at $\infty$ is

$$
g_{E}(z):=\log \frac{1}{\operatorname{cap} E}+\int_{E} \log |z-t| d \mu(t),
$$

where $\mu$ is the so-called equilibrium measure of $E$. This $\mu$ is a probability measure supported on the outer boundary $\partial E$ of $E$. If $E$ is a set regular with respect to the Dirichlet problem (as our lemniscates certainly are), then $g_{E}(z)=0, z \in \partial E$, and $g_{E}$ is harmonic in $\mathbb{C} \backslash E$, with

$$
g_{E}(z)-\log |z| \rightarrow \log \frac{1}{\operatorname{cap} E},|z| \rightarrow \infty .
$$

All this may be found in [9], [10], [12]. 
Proof of (1.8) - (1.10) of Theorem 1.1. Let $P(z)$ be a polynomial of degree $\leq n$, normalized by $(1.5)$. Let $E:=E(P ; r ; \epsilon)$. As the ball $\{z:|z| \leq r\}$ has cap $r$, we need prove (1.8) only for $\epsilon \leq \frac{1}{2}$. The well-known Walsh-Bernstein Lemma states that

$$
|P(z)| \leq\|P\|_{L_{\infty}(E)}\left(e^{g_{E}(z)}\right)^{n}, z \in \mathbb{C} \backslash E .
$$

Using our normalization, we obtain

$$
1=\|P\|_{L_{\infty}(|z| \leq r)} \leq \epsilon^{n} \exp \left(n \sup \left\{g_{E}(z):|z| \leq r, z \notin E\right\}\right) .
$$

But $\mu$ is a probability measure on $E \subset\{t:|t| \leq r\}$ so, for $|z| \leq r, z \notin E$,

$$
g_{E}(z) \leq \log \frac{1}{\operatorname{cap} E}+\int_{E} \log (2 r) d \mu(t)=\log \left(\frac{2 r}{\operatorname{cap} E}\right) .
$$

Thus

$$
1 \leq\left(\frac{\epsilon 2 r}{\operatorname{cap} E}\right)^{n}
$$

from which (1.8) follows. The well-known inequalities [7, pp. 300-302]

$$
\begin{aligned}
m_{2}(E) & \leq \pi(\operatorname{cap} E)^{2} ; \\
h_{1}(L \cap E) & \leq 4 \operatorname{cap} E
\end{aligned}
$$

then give (1.9) and (1.10).

Proof of (1.11) - (1.13). Fix $0<a<r$, and let

$$
P_{1}(z):=\left(\frac{z+a}{r+a}\right)^{n}
$$

Then $P_{1}$ satisfies (1.5), and

$$
\left|P_{1}(z)\right| \leq \epsilon^{n} \Leftrightarrow|z+a| \leq \epsilon(r+a) .
$$

We see that for

$$
0<\epsilon \leq \frac{r-a}{r+a},
$$

the whole of the ball centre $-a$, radius $\epsilon(r+a)$, is contained in $\{z:|z| \leq r\}$. Thus for such $\epsilon$,

$$
E\left(P_{1} ; r ; \epsilon\right)=\{z:|z+a| \leq \epsilon(r+a)\},
$$

so

$$
\begin{aligned}
& \operatorname{cap}\left(E\left(P_{1} ; r ; \epsilon\right)\right)=\epsilon(r+a) ; \\
& m_{2}\left(E\left(P_{1} ; r ; \epsilon\right)\right)=\pi(\epsilon(r+a))^{2} .
\end{aligned}
$$


Hence

$$
\begin{aligned}
& \sup _{P, \epsilon} \operatorname{cap}(E(P ; r ; \epsilon)) / \epsilon \geq r+a ; \\
& \sup _{P, \epsilon} m_{2}(E(P ; r ; \epsilon)) / \epsilon^{2} \geq \pi(r+a)^{2} .
\end{aligned}
$$

Since we may make $a$ arbitrarily close to $r$, we obtain $(1.11)-(1.12)$. The proof of (1.13) is a little more complicated: Let $0<a<r$, and $T_{n}(x)=\cos (n \arccos x)$ denote the usual Chebyshev polynomial for $[-1,1]$, and for small $\delta>0$ (actually $\delta<r-a$ will do), let

$$
P_{1}(z):=T_{n}\left(\frac{z+a}{\delta}\right) /\left\|T_{n}\left(\frac{u+a}{\delta}\right)\right\|_{L_{\infty}(|u| \leq r)} .
$$

Then $P_{1}$ satisfies (1.5). Moreover, with

$$
\epsilon:=\left\|T_{n}\left(\frac{u+a}{\delta}\right)\right\|_{L_{\infty}(|u| \leq r)}^{-1 / n}
$$

we see that

$$
E\left(P_{1} ; r ; \epsilon\right)=\left\{z:|z| \leq r \text { and }\left|T_{n}\left(\frac{z+a}{\delta}\right)\right| \leq 1\right\}=[-a-\delta,-a+\delta]
$$

SO

$$
h_{1}\left(E\left(P_{1} ; r ; \epsilon\right)\right) / \epsilon=2 \delta T_{n}\left(\frac{r+a}{\delta}\right)^{1 / n} .
$$

Now $T_{n}$ has leading coefficient $2^{n-1}$, so behaves for large $x$ like $2^{n-1} x^{n}$. Then given $\eta \in(0,1)$, we have if $\delta$ is small enough,

$$
h_{1}\left(E\left(P_{1} ; r ; \epsilon\right)\right) / \epsilon \geq 2 \delta \eta 2^{1-1 / n}\left(\frac{r+a}{\delta}\right)=4(r+a) 2^{-1 / n} \eta
$$

Since $a$ may be chosen arbitrarily close to $r$, and $\eta$ may be chosen arbitrarily close to 1 , we obtain (1.13).

Proof of (1.14) of Theorem 1.1. This follows from (1.8) and the estimate [12, p.203] $h_{\alpha}(E) \leq 18(2 \operatorname{cap} E)^{\alpha}$.

\section{§3. Proof of Theorems 1.2 And 1.3}

We begin with a lemma on the maximum of a polynomial along a slice:

Lemma 3.1. Let $P\left(z_{1}, z_{2}, \ldots, z_{k}\right)$ be a polynomial of degree $\leq n$ in each variable that satisfies (1.20). For fixed $z_{1}$, let

$$
M\left(z_{1}\right):=\max \left\{\left|P\left(z_{1}, z_{2}, z_{3}, \ldots, z_{k}\right)\right|:\left|z_{j}\right| \leq r, 2 \leq j \leq k\right\}
$$


and let

$$
\mathcal{E}:=\left\{z_{1}:\left|z_{1}\right| \leq r \text { and } M\left(z_{1}\right) \leq \epsilon^{n}\right\}
$$

Then

$$
\operatorname{cap}(\mathcal{E}) \leq 2 r \epsilon ; \quad m_{2}(\mathcal{E}) \leq \pi(2 r \epsilon)^{2} .
$$

Proof. Choose $z_{j}, 2 \leq j \leq k$, such that each $\left|z_{j}\right| \leq r$ and

$$
\max \left\{\left|P\left(u, z_{1}, z_{2}, \ldots, z_{k}\right)\right|:|u| \leq r\right\}=1 .
$$

This is possible by our normalization (1.20). With these variables chosen, $Q\left(z_{1}\right):=$ $P\left(z_{1}, z_{2}, \ldots, z_{k}\right)$ is a polynomial of degree $\leq n$ in $z_{1}$ with

$$
\left|Q\left(z_{1}\right)\right|:=\left|P\left(z_{1}, z_{2}, \ldots, z_{k}\right)\right| \leq M\left(z_{1}\right) \leq \epsilon^{n}, z_{1} \in \mathcal{E},
$$

and

$$
\|Q\|_{L_{\infty}\left(\left|z_{1}\right| \leq r\right)}=1
$$

Then

$$
\mathcal{E} \subset E(Q ; r ; \epsilon),
$$

so

$$
\operatorname{cap}(\mathcal{E}) \leq \operatorname{cap}(E(Q ; r ; \epsilon)) \leq 2 r \epsilon,
$$

by Theorem 1.1. Then (2.2) gives the estimate for $m_{2}(\mathcal{E})$.

Proof of Theorem 1.2. We do this by induction on $k$. We can assume that $\epsilon<1$, since if $\epsilon \geq 1$, then $E(P ; r ; \epsilon)$ is all of the polydisc $\mathcal{P}:=\left\{\left|z_{j}\right| \leq r, 1 \leq j \leq k\right\}$, so has $m_{2 k}$ measure $\left(\pi r^{2}\right)^{k}$ and (1.22) is immediate.

(1.22) is true for $k=1$. For $k=1$, the result follows from Theorem 1.1.

Assume (1.22) is true for $k-1$, and prove true for $k$. Let us write

$$
\underline{z}^{\prime}=\left(z_{2}, z_{3}, \ldots, z_{k}\right) ; \underline{z}:=\left(z_{1}, \underline{z}^{\prime}\right)=\left(z_{1}, z_{2}, \ldots, z_{k}\right) .
$$

We let $\mathcal{P}$ be as above and we let $\mathcal{P}^{\prime}$ denote the polydisc $\left\{\underline{z}^{\prime}:\left|z_{j}\right| \leq r, 2 \leq j \leq k\right\}$. For $z_{1}$ fixed, let $M\left(z_{1}\right)$ denote the maximum modulus of $P(\underline{z})$ along a slice, as in (3.1). Note that for fixed $z_{1}$,

$$
Q\left(\underline{z}^{\prime}\right):=P(\underline{z}) / M\left(z_{1}\right)
$$

has

$$
\max \left\{\left|Q\left(\underline{z}^{\prime}\right)\right|: \underline{z} \in \mathcal{P}^{\prime}\right\}=1 .
$$

By our induction step (recall $z_{1}$ is fixed),

$$
\begin{aligned}
m_{2(k-1)} & \left\{\underline{z}^{\prime} \in \mathcal{P}^{\prime}:|P(\underline{z})| \leq \epsilon^{n}\right\} \\
& =m_{2(k-1)}\left\{\underline{z}^{\prime} \in \mathcal{P}^{\prime}:\left|Q\left(\underline{z}^{\prime}\right)\right| \leq \epsilon^{n} / M\left(z_{1}\right)\right\} \\
& \leq\left(16 \pi r^{2}\right)^{k-1} \frac{\epsilon^{2}}{M\left(z_{1}\right)^{2 / n}} \max \left\{1, \log _{2} \frac{2^{k-2} M\left(z_{1}\right)^{1 / n}}{\epsilon}\right\}^{k-2} .
\end{aligned}
$$


Let us set

$$
\begin{aligned}
\mathcal{E}_{-1}: & =\left\{z_{1}:\left|z_{1}\right| \leq r \text { and } M\left(z_{1}\right) \leq \epsilon^{n}\right\} \\
\mathcal{E}_{j}: & =\left\{z_{1}:\left|z_{1}\right| \leq r \text { and }\left(2^{j} \epsilon\right)^{n}<M\left(z_{1}\right) \leq\left(2^{j+1} \epsilon\right)^{n}\right\}, j \geq 0 .
\end{aligned}
$$

Since $M\left(z_{1}\right) \leq 1, \mathcal{E}_{j}$ is empty if

$$
2^{j} \epsilon \geq 1 \Leftrightarrow j \geq \log _{2} \frac{1}{\epsilon}
$$

By Lemma 3.1,

$$
\begin{aligned}
m_{2}\left(\mathcal{E}_{-1}\right) & \leq \pi(2 r \epsilon)^{2} \\
m_{2}\left(\mathcal{E}_{j}\right) & \leq \pi\left(2 r 2^{j+1} \epsilon\right)^{2} .
\end{aligned}
$$

Then by (3.4), if $\ell=$ greatest integer $\leq \log _{2} \frac{1}{\epsilon}-1$,

$$
\begin{aligned}
m_{2 k}(E(P ; r ; \epsilon))= & \int_{\left|z_{1}\right| \leq r} m_{2(k-1)}\left\{\underline{z}^{\prime} \in \mathcal{P}^{\prime}:|P(\underline{z})| \leq \epsilon^{n}\right\} d m_{2}\left(z_{1}\right) \\
\leq & \int_{\left|z_{1}\right| \leq r} \min \left\{\left(\pi r^{2}\right)^{k-1},\left(16 \pi r^{2}\right)^{k-1} \frac{\epsilon^{2}}{M\left(z_{1}\right)^{2 / n}}\right. \\
& \left.\times \max \left\{1, \log _{2} \frac{2^{k-2} M\left(z_{1}\right)^{1 / n}}{\epsilon}\right\}^{k-2}\right\} d m_{2}\left(z_{1}\right) \\
\leq & \left(\pi r^{2}\right)^{k-1}\left[\int d m_{2}\left(z_{1}\right)\right. \\
\leq & \left(\pi r^{2}\right)^{k}\left[4 \epsilon^{2}+16^{k-1} 16 \epsilon^{2} \sum_{j=0}^{\ell}\left(\log _{2}\left[2^{k-2} / \epsilon\right]\right)^{k-2}\right] \\
& +\sum_{j=0} \int_{\mathcal{E}_{j}} \frac{16^{k-1} \epsilon^{2}\left(2^{j} \epsilon\right)^{2}\left(\log _{2}\left[2^{k-2} 2^{j+1}\right]\right)^{k-2} d m_{2}\left(z_{1}\right)}{\left.\leq \pi r^{2}\right)^{k} \epsilon^{2}\left[1+\left(\log _{2}\left[2^{k-2} / \epsilon\right]\right)^{k-1}\right],} \\
\leq & (16)
\end{aligned}
$$

where we have used our choice of $\ell$, and also $\epsilon \leq 1$. Finally,

$$
\left[1+\left(\log _{2}\left[2^{k-2} / \epsilon\right]\right)^{k-1}\right] \leq\left[1+\log _{2}\left[2^{k-2} / \epsilon\right]\right]^{k-1}=\left[\log _{2}\left[2^{k-1} / \epsilon\right]\right]^{k-1} .
$$

So we have completed the proof for $k$.

Proof of (1.26) of Theorem 1.3. We keep the notation $\underline{z}, \underline{z}^{\prime}, \mathcal{P}, \mathcal{P}^{\prime}$ from the previous proof. We can assume $\epsilon \leq 1$, for if $\epsilon>1$, then $E(P ; r ; \epsilon)=\mathcal{P}$, and $\operatorname{ascap}^{(k)}(\mathcal{P})=r^{k}$ (this is easily proved by induction on $k$ ), (1.26) is immediate. So we assume $\epsilon<1$, and proceed by induction on $k$ : 
(1.26) is true for $k=1$. This follows directly from Theorem 1.1, with $C_{1}=2$.

Assume (1.26) true for $k-1$, some $k \geq 2$. Let $P\left(z_{1}, z_{2}, \ldots, z_{k}\right)$ be of degree $\leq n$ in each variable, normalized by (1.20). Let $M\left(z_{1}\right)$ be the maximum modulus along a slice, as in (3.1). By definition,

$\operatorname{cap}^{(k)}(E(P ; r ; \epsilon))=\int_{0}^{\infty} \operatorname{cap}\left\{z_{1}:\left|z_{1}\right| \leq r\right.$ and $\left.\operatorname{cap}^{(k-1)}\left\{\underline{z}^{\prime}: \underline{z} \in E(P ; r ; \epsilon)\right\}>s\right\} d s$.

By our induction hypothesis, namely (1.26) for $k-1$,

$$
\begin{aligned}
\operatorname{cap}^{(k-1)}\left\{\underline{z}^{\prime}: \underline{z} \in E(P ; r ; \epsilon)\right\} \\
\quad=\operatorname{cap}^{(k-1)}\left\{\underline{z}^{\prime} \in \mathcal{P}^{\prime}:|P(\underline{z})| / M\left(z_{1}\right) \leq \epsilon^{n} / M\left(z_{1}\right)\right\} \\
\quad \leq C_{1} r^{k-1} \frac{\epsilon}{M\left(z_{1}\right)^{1 / n}} \max \left\{1, \log _{2} \frac{M\left(z_{1}\right)^{1 / n}}{\epsilon}\right\}^{k-2} .
\end{aligned}
$$

Moreover, this set is contained in $\mathcal{P}^{\prime}$, so has cap ${ }^{(k-1)} \leq r^{k-1}$. Thus

$$
\operatorname{cap}^{(k-1)}\left\{\underline{z}^{\prime}: \underline{z} \in E(P ; r ; \epsilon)\right\} \leq r^{k-1} F\left(\epsilon / M\left(z_{1}\right)^{1 / n}\right),
$$

where

$$
F(u):=\min \left\{1, C_{1} u \max \left\{1, \log _{2} \frac{1}{u}\right\}^{k-2}\right\}
$$

So,

$$
\begin{aligned}
\operatorname{cap}^{(k)}(E(P ; r ; \epsilon)) & \leq \int_{0}^{r^{k-1}} \operatorname{cap}\left\{z_{1}:\left|z_{1}\right| \leq r \text { and } r^{k-1} F\left(\epsilon / M\left(z_{1}\right)^{1 / n}\right)>s\right\} d s \\
& =r^{k-1} \int_{0}^{1} \operatorname{cap}\left\{z_{1}:\left|z_{1}\right| \leq r \text { and } F\left(\epsilon / M\left(z_{1}\right)^{1 / n}\right)>t\right\} d t .
\end{aligned}
$$

We see that there exists $C_{2}>0$ such that for $t \in(0,1]$,

$$
F(u)>t \Rightarrow u>C_{2} t \max \left\{1, \log _{2} \frac{1}{t}\right\}^{-(k-2)}
$$

Hence

$$
F\left(\epsilon / M\left(z_{1}\right)^{1 / n}\right)>t \Rightarrow M\left(z_{1}\right)<\left(\frac{\epsilon \max \left\{1, \log _{2} \frac{1}{t}\right\}^{k-2}}{C_{2} t}\right)^{n}
$$


By Lemma 3.1, the set of $\left|z_{1}\right| \leq r$ with $M\left(z_{1}\right)$ satisfying this inequality has cap at most

$$
2 r \frac{\epsilon \max \left\{1, \log _{2} \frac{1}{t}\right\}^{k-2}}{C_{2} t},
$$

and also has cap $\leq r$. So (3.5) gives

$$
\begin{aligned}
\operatorname{cap}^{(k)}(E(P ; r ; \epsilon)) & \leq r^{k} \int_{0}^{1} \min \left\{1,2 \frac{\epsilon \max \left\{1, \log _{2} \frac{1}{t}\right\}^{k-2}}{C_{2} t}\right\} d t \\
& \leq r^{k}\left\{\int_{0}^{\epsilon} d t+2 C_{2}^{-1} \epsilon \max \left\{1, \log _{2} \frac{1}{\epsilon}\right\}^{k-2} \int_{\epsilon}^{1} \frac{d t}{t}\right\} \\
& \leq C_{3} r^{k} \epsilon \max \left\{1, \log _{2} \frac{1}{\epsilon}\right\}^{k-1}
\end{aligned}
$$

where $C_{3}$ depends only on $k$.

Proof of (1.27) and (1.28) of Theorem 1.3. We let $\underline{z}=\left(z_{1}, z_{2}, \ldots, z_{k}\right)$ and

$$
\|\underline{z}\|:=\left\{\sum_{j=1}^{k}\left|z_{j}\right|^{2}\right\}^{1 / 2} .
$$

We shall use the following properties of a unitary matrix $A$ : The inverse $A^{-1}$ is also unitary, and $[19$, p.74]

$$
\|A \underline{z}\|=\|\underline{z}\| .
$$

Now if $P(\underline{z})$ is of degree $\leq n$ in each variable, and $Q(\underline{z}):=P\left(A^{-1} \underline{z}\right)$, then $Q(\underline{z})$ is of degree $\leq k n$ in each variable. If in addition $P$ is of total degree $\leq n$, then we see that $Q(\underline{z})$ is of degree $\leq n$ in each variable. Moreover, setting $\underline{w}=A \underline{z}$, we see that

$$
\begin{aligned}
A(E(P ; r ; \epsilon)) & =\left\{A \underline{z} \text { : each }\left|z_{j}\right| \leq r \text { and }|P(\underline{z})| \leq \epsilon^{n}\right\} \\
& =\left\{\underline{w}: \text { each }\left|\left(A^{-1} \underline{w}\right)_{j}\right| \leq r \text { and }|Q(\underline{w})| \leq \epsilon^{n}\right\} .
\end{aligned}
$$

Here, of course, $\left(A^{-1} \underline{w}\right)_{j}$ denotes the $j$ th component of the $k$-vector $A^{-1} \underline{w}$. Then $\forall j$

$$
\left|w_{j}\right| \leq\|\underline{w}\|=\left\|A^{-1} \underline{w}\right\| \leq \sqrt{k} \max _{j}\left|\left(A^{-1} \underline{w}\right)_{j}\right| \leq \sqrt{k} r .
$$

Thus, regarding $Q$ as a polynomial of degree $\leq k n$ in each variable,

$$
A(E(P ; r ; \epsilon)) \subseteq E\left(Q ; \sqrt{k} r ; \epsilon^{1 / k}\right) .
$$

(If $P$ is of total degree $\leq n$, we can regard $Q$ as a polynomial of degree $\leq n$ in each variable, and replace $\epsilon^{1 / k}$ by $\epsilon$.) Next, if $\underline{w}=A \underline{z}$, and each $\left|z_{j}\right| \leq r$, we have shown each $\left|w_{j}\right| \leq \sqrt{k} r$, so

$$
\max \left\{|Q(\underline{w})|: \text { each }\left|w_{j}\right| \leq \sqrt{k} r\right\} \geq \max \left\{|P(\underline{z})|: \text { each }\left|z_{j}\right| \leq r\right\}=1 .
$$


Thus our (1.26) applied to $Q$ gives

$$
\begin{aligned}
\operatorname{cap}^{(k)}[A(E(P ; r ; \epsilon))] & \leq \operatorname{cap}^{(k)}\left[E\left(Q ; \sqrt{k} r ; \epsilon^{1 / k}\right)\right] \\
& \leq C_{1} \sqrt{k^{k}} r^{k} \epsilon^{1 / k} \max \left\{1, \frac{1}{k} \log _{2} \frac{1}{\epsilon}\right\}^{k-1} .
\end{aligned}
$$

So we have (1.27). When $P$ has total degree $\leq n$, we can replace $\epsilon^{1 / k}$ by $\epsilon$ and hence obtain (1.28).

\section{NOTE ADDED IN PROOF}

After this paper was accepted, Prof. Tom Bloom of the University of Toronto provided the authors with related references for the classical capacities in $\mathbb{C}^{k}$ :

1. N. Levenberg and B. Taylor, Comparison of capacities in $\mathbb{C}^{n}$, Lecture Notes in Math., vol. 1094, Springer, Berlin, 1984, pp. 152-162.

2. S. Kolodziej, The logarithmic capacity in $\mathbb{C}^{n}$, Ann. Polon. Math. 48 (1988), 253-267.

\section{REFERENCES}

1. G.A. Baker, Essentials of Padé Approximants, Academic Press, New York, 1975. MR 56:12710

2. G.A. Baker and P.R. Graves-Morris, Padé Approximants, Part 1: Basic Theory, Encyclopaedia of Mathematics and its Applications, Vol. 13, Addison-Wesley, Reading, MA, 1981. MR 83a:41009a

3. E. Bedford and B.A. Taylor,, The Complex Equilibrium Measure of a Symmetric Convex Set in $\mathbb{R}^{n}$, Trans. Amer. Math. Soc. 294 (1986), 705-717. MR 87f:32039

4. P. Borwein and T. Erdelyi, Polynomials and Polynomial Inequalities, Book (to appear).

5. A. Cuyt, Multivariate Padé Approximants Revisited, BIT 26 (1986), 71-79. MR 87f:41031

6. U. Cegrell, Capacities in Complex Analysis, Aspects of Mathematics, Vol. 14, Vieweg, Braunschweig, 1988. MR 89m:32001

7. G.M. Goluzin, Geometric Theory of Functions of a Complex Variable, Translations of Math. Monographs, Vol. 26, American Mathematical Society, Providence, 1969. MR 40:308

8. A.A. Goncar, Local Condition for the Single-Valuedness of Analytic Functions of Several Variables, Math. USSR. Sbornik 22 (1974), 305-322. MR 58:28632

9. W.K. Hayman and P.B. Kennedy, Subharmonic Functions, Vol. 1,, Academic Press, London, 1976. MR 57:665

10. E. Hille,, Analytic Function Theory, Vol. 2, Chelsea, New York, 1987. MR 34:1490 (earlier ed.)

11. J. Karlsson and H. Wallin, Rational Approximation by an Interpolation Procedure in Several Variables, (in) Padé and Rational Approximation: Theory and Applications (eds. E.B. Saff and R.S. Varga), Academic Press, New York, 1977, pp. 83-100. MR 58:1877

12. N.S. Landkof, Foundations of Modern Potential Theory, Grundlehren der Mathematischen Wissenchaften, Vol. 180, Springer, Berlin, 1972. MR 50:2520

13. J. Nuttall, The Convergence of Padé Approximants of Meromorphic Functions, J. Math. Anal. Applns. 31 (1970), 147-153. MR 44:5477

14. B. Paneah, On a Lower Bound for the Absolute Value of a Polynomial of Several Variables, J. Approx. Theory 78 (1994), 402-409. MR 95j:32020

15. C. Pommerenke, Padé Approximants and Convergence in Capacity, J. Math. Anal. Applns. 41 (1973), 775-780. MR 48:6432

16. C.A. Rogers, Hausdorff Measures, Cambridge University Press, Cambridge, 1970. MR 43:7576

17. A. Sadullaev, Plurisubharmonic Measures and Capacities on Complex Manifolds, Russian Math. Surveys 36 (1981), 61-119. MR 83c:32026 (Russian original)

18. M. Schiffer and J. Siciak, Transfinite Diameter and Analytic Continuation of Functions of Two Complex Variables, (in) Studies in Math. Anal. and Related Topics, Stanford University Press, Stanford, 1962, pp. 341-358. MR 27:342 
19. G.W. Stewart, and J-G. Sun, Matrix Perturbation Theory, Academic Press, London, 1990. MR 92a:65017

20. V.P. Zaharjuta, Transfinite Diameter, Chebyshev Constants, and Capacity for Compacta in $\mathbb{C}^{n}$, Math. USSR. Sbornik 25 (1975), 350-364. MR 58:6342

(A. Cuyt) Department of Mathematics, UiA, University of Antwerp, UniversiteitSPLEIN 1, B2610 WilRIJK, BELGIUM

E-mail address: CUYT@WINS.UIA.AC.BE

(K. Driver and D. S. Lubinsky) Department of Mathematics, Witwatersrand University, P.O. Wits 2050, South Africa

E-mail address: K. Driver: 036KaD@COSMOS.WITS.AC.ZA

E-mail address: D. Lubinsky: 036DSL@COSMOS.WITS.AC.ZA 